\title{
USO DA TECNOLOGIA BIM EM ESCRITÓRIOS E EMPRESAS DE ENGENHARIA E ARQUITETURA NAS REGIÕES BRASILEIRAS
}

\author{
Tayla Carolina da Silva - taylac_silva@hotmail.com \\ Universidade Tecnológica Federal do Paraná \\ Apucarana - Paraná \\ Mileni Cristina da Silva-contato@milenisilva.eng.br \\ Universidade Tecnológica Federal do Paraná \\ Apucarana - Paraná
}

\section{Resumo}

Este artigo visa diagnosticar a disseminação da tecnologia BIM e a perspectiva de futuro entre profissionais e estudantes de engenharia e arquitetura tanto para a elaboração, quanto para a compatibilização, planejamento, gerenciamento e controle de projetos no segmento da construção civil, por meio de uma pesquisa de abrangência nacional, voluntária e divulgada por meios eletrônicos. Através do levantamento dos dados obtidos nas diferentes regiões do país, com faixa etária e tempo de atuação variáveis, e com diferentes esferas de atuação dentro da área de arquitetura, engenharia e construção, torna-se possível realizar uma análise das informações obtidas com o objetivo de apresentar os obstáculos que podem estar diretamente ou indiretamente ligadas ao fato de o escritório ou empresa fazer uso ou não do BIM no processo de desenvolvimento de seus projetos. Os principais motivos encontrados entre os profissionais que ainda não fazem uso de softwares BIM foram pelo valor que é necessário investir nestes softwares, e também pelo tempo que é necessário para treinar os funcionários. A obrigatoriedade de utilização do BIM para as licitações de obras públicas federais, que está próxima de acontecer, acaba induzindo o mercado da construção civil a se modernizar como um todo. Estima-se que em um curto espaço de tempo, as empresas e profissionais de projetos que não adotarem o BIM em seus processos de trabalho poderão perder competitividade e, aos poucos, serem excluídos do mercado.

Palavras-chave: Tecnologia BIM. Projetos. Modernização. Construção civil.

\section{USE OF BIM TECHNOLOGY AMONG BRAZILIAN OFFICES AND COMPANIES OF ENGINEERING AND ARCHITECTURE}

\section{Abstract:}

The main objective of this article is to diagnose the current dissemination of BIM technology and the future perspective among professionals and students of engineering and architecture, through a voluntary research released by electronic means to all regions of Brazil. It was considered the utilization for projects development, for compatibilization, planning, management and control of projects in the civil construction segment. Within the data survey obtained in different regions of the country, with variable age range and time of activity, and with different classes of activity within the area of architecture, engineering and construction, it is possible to carry out an analysis of the information obtained by reporting the obstacles that may be directly or indirectly linked to the fact that the office or company uses or not BIM technology in the process of developing its projects. The main reasons found among professionals who do not yet use BIM software were for the amount that is necessary to invest in these software programs, and also for the time that is necessary to train employees. The mandatory use of BIM for bids for federal public works, which is close to happening, ends up inducing the civil construction market to modernize as a whole. In a short piece of time, companies and project professionals who do not adopt BIM in their work processes may lose competitiveness and, little by little, be excluded from the market.

Keywords: BIM Technology. Projects. Upgrading. Civil construction. 


\section{INTRODUÇÃ̃O}

A modernização no setor da construção civil vem acontecendo tanto na elaboração de projetos, quanto no planejamento, gestão e controle das obras civis. Desde obras mais simples até as mais complexas, todas possuem características próprias que podem dificultar a compatibilização dos diferentes projetos que compõem uma mesma edificação. Para otimização destes processos, diversos profissionais buscam obter assistência de ferramentas computacionais, como softwares e aplicativos, a fim de garantir resultados de maior precisão.

Os softwares que estão atuando no mercado brasileiro há mais tempo oferecem o chamado desenho assistido por computador (Computer-Aided Design - CAD) (RIBEIRO; PEREIRA, 2015), com a modelagem realizada com linhas (1D) e planos (2D). Dessa forma, os softwares com tecnologia BIM são considerados inovadores por permitirem a comunicação e interoperabilidade entre os integrantes do projeto em tempo real, além de ser composto por até sete dimensões sendo elas: representação tridimensional (3D), análise de tempo (4D), análise de quantidades e custos (5D), tecnologia de sustentabilidade (6D), e gestão da construção (7D) (BIBLUS, 2018).

De acordo com Pereira e Ribeiro (2015), existem diferentes estágios ou níveis de maturidade no uso de ferramentas computacionais para o desenvolvimento de projetos. Dentre as que não utilizam a tecnologia BIM, existem as que utilizam apenas ferramentas bidimensionais como linhas, arcos e textos, e também as que podem ser utilizadas para criar modelos tridimensionais. Já as ferramentas que fazem uso do BIM, se dividem entre as que utilizam parametrização dos objetos e desenvolvimento de projeto colaborativo, e as que integram e gerenciam todo o ciclo de vida da edificação.

Portanto, as dimensões do BIM vão além das dimensões já existentes e muito utilizadas no campo da construção civil como o $2 \mathrm{D}$ e até mesmo a modelagem $3 \mathrm{D}$, que já ocorre há alguns anos, com o desenvolvimento de maquetes virtuais. O modelo 3D em BIM é uma evolução da maquete virtual, pois apresenta informações relativas a índices e especificações técnicas dos diversos elementos que compõem a edificação. Na figura 1, tem-se uma representação das dimensões oferecidas pela tecnologia BIM.

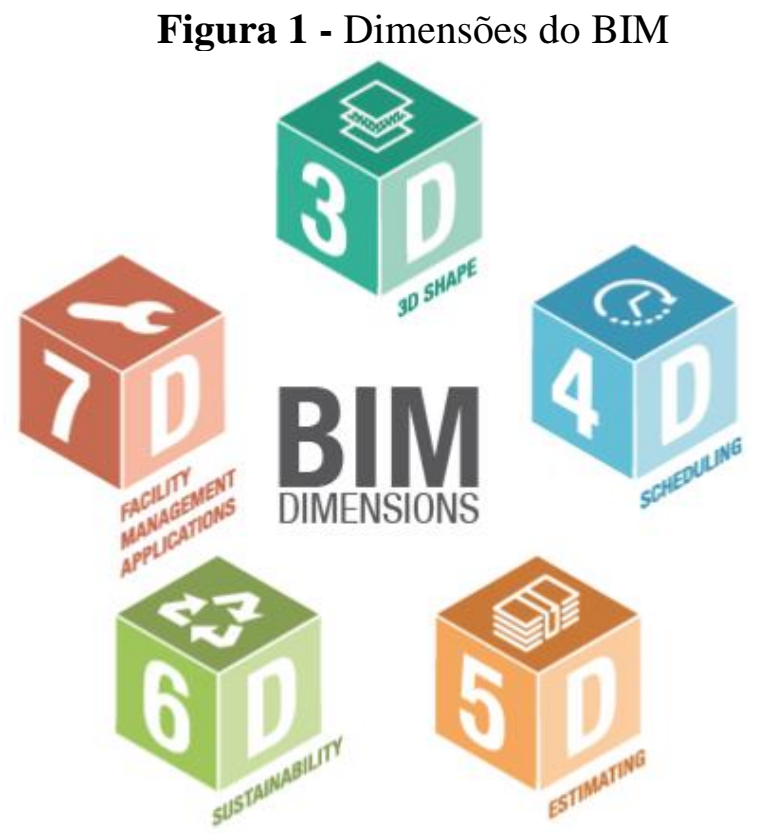

Fonte - (BIBLUS, 2018)

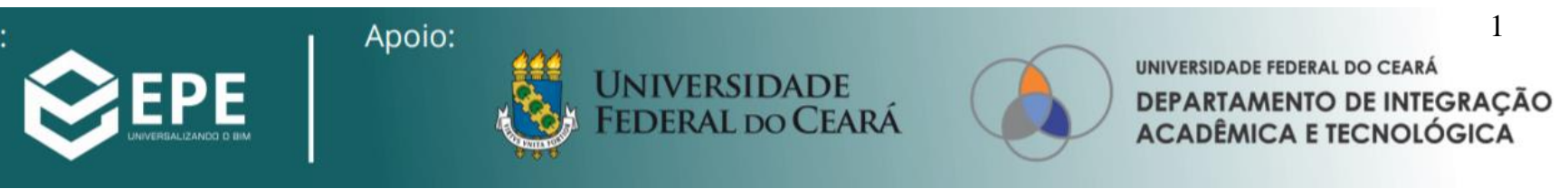


Em 2018, o governo federal anunciou por meio do decreto ${ }^{\circ} 9.377$ (BRASIL, 2018), um comitê Gestor da Estratégia BIM BR, que tem por finalidade promover um ambiente adequado ao investimento em tecnologia BIM e disseminação do uso deste, por todo o país. O decreto foi revogado por uma nova versão de $n^{\circ} 9.983$ em 2019. A Estratégia Nacional de Disseminação do Building Information Modelling (Estratégia BIM BR) é integrada por representantes de nove ministérios, os quais ficam responsáveis por sua implantação, gerenciamento e supervisão do funcionamento e avanço deste processo. Em situações em que não houver o cumprimento das metas, os representantes viabilizarão soluções e aprimoramentos. Esta estratégia nacional tem o intuito de adequar a atuação dos agentes públicos e privados, aumentando o índice de precisão de cálculos, eficiência ao longo das etapas da obra e melhoramento da competitividade no mercado da construção civil (ECONOMIA, 2018).

Em 2020, através do decreto $\mathrm{n}^{\circ} 10.306$ (BRASIL, 2020), a Estratégia BIM BR estabeleceu as fases de implementação do BIM na execução direta ou indireta de obras e serviços de engenharia realizada pelos órgãos e pelas entidades da administração pública federal. Na primeira fase, a partir de janeiro de 2021, a exigência de BIM se dará na elaboração de modelos para a Arquitetura e Engenharia nas disciplinas de estrutura, hidráulica, AVAC e elétrica na detecção de interferências, na extração de quantitativos e na geração de documentação gráfica a partir desses modelos. Na segunda fase, a partir de janeiro de 2024, os modelos deverão contemplar algumas etapas que envolvem a obra, como o planejamento da execução da obra, na orçamentação e na atualização dos modelos e de suas informações como construído ("as built"), além das exigências da primeira fase. E na terceira fase, a partir de janeiro de 2028, passará a abranger todo o ciclo de vida da obra ao considerar atividades do pós-obra. Será aplicado, no mínimo, nas construções novas, reformas, ampliações ou reabilitações, quando consideradas de média ou grande relevância, nos usos previstos na primeira e na segunda fases e, além disso, nos serviços de gerenciamento e de manutenção do empreendimento após sua conclusão.

A implantação da tecnologia BIM é crescente no Brasil e também no contexto mundial, e tem provocado uma revolução no ramo da construção civil, atingindo toda a cadeia produtiva das áreas de arquitetura, engenharia e construção (AEC). Nas universidades, também existe grande interesse no aprendizado da metodologia, seja por iniciativa de professores ou pela demanda dos próprios alunos. Estima-se que em um curto espaço de tempo, as empresas e profissionais de projetos que não adotarem o BIM em seus processos de trabalho poderão perder competitividade e, aos poucos, serem excluídos do mercado (SEIL, 2018)

\section{OBJETIVOS}

Este estudo tem como objetivo principal diagnosticar a atual disseminação da tecnologia BIM e a perspectiva de futuro entre profissionais de engenharia e arquitetura tanto para a elaboração, quanto para a compatibilização, planejamento, gerenciamento e controle de projetos no segmento da construção civil, por meio de uma pesquisa de abrangência nacional, que obteve participação voluntária, entre profissionais e estudantes do setor, de forma amostral em cada região brasileira.

Através do levantamento dos dados obtidos nas diferentes regiões do país, com faixa etária, tempo de atuação variáveis e com diferentes esferas de atuação dentro da área de arquitetura, engenharia e construção, torna-se possível realizar uma análise das informações obtidas com o objetivo de avaliar os obstáculos que podem estar diretamente ou indiretamente ligadas ao fato de o escritório ou empresa fazer uso ou não do BIM no processo de desenvolvimento e gerenciamento de projetos. 


\section{METODOLOGIA}

Para realizar esta pesquisa, foi elaborado um formulário online utilizando a plataforma do Google e divulgado em redes sociais (Instagram, Facebook, Linkedin) e grupos de WhatsApp e Telegram, durante o mês de agosto de 2020. O formulário foi distribuído com livre acesso e houve a participação voluntária de 170 estudantes e profissionais de engenharia e arquitetura, distribuídos pelas regiões brasileiras.

Através dos dados da pesquisa, foi possível filtrar não só a localização onde o entrevistado reside, mas também outras características como gênero, faixa etária, nível de escolaridade, renda familiar e profissão. O formulário foi realizado com o propósito de entrevistar os atuais e futuros profissionais integrantes do mercado nacional da construção civil, para conhecer a atual aplicação da tecnologia BIM na empresa ou escritório em que o entrevistado desempenha sua função.

A primeira parte do questionário foi elaborado com o objetivo de identificar o entrevistado quanto à região em que reside, gênero, faixa etária, escolaridade, profissão, tempo de atuação no cargo, porte da empresa ou escritório no qual trabalha, a função desempenhada e a atual situação socioeconômica. Esses questionamentos refletem de forma ampla, como o mercado de trabalho no setor da construção civil está estruturado quanto ao tempo de atuação e a qualificação dos profissionais.

A segunda parte da entrevista, cerca de $50 \%$ do formulário, foi exclusivamente atribuída aos conhecimentos e utilizações de softwares BIM, indagando se os profissionais sabem o que é a tecnologia BIM, se o escritório que trabalham fazem uso desses softwares tanto para projetos quanto para o gerenciamento de obras e quais softwares são utilizados. Também foi questionado quais softwares são utilizados na rotina do escritório, com ou sem a tecnologia BIM e o porquê de ainda não fazer uso de softwares BIM.

A pesquisa sugere algumas causas pelas quais os escritórios de projetos ainda possuem resistência em aderir os softwares BIM, como sugestão foram destacadas: o alto investimento para aderir um software BIM, a dificuldade em se organizar com tempo para a capacitação dos funcionários, a comodidade por ter funcionários tendo um bom rendimento usando o software que está acostumado, entre outros obstáculos, assim encontrado pelos profissionais para permanecerem no mesmo nível de tecnologia em que estão acostumados ou que possam estar, mediante situações socioeconômicas do escritório.

\section{RESULTADOS}

O formulário atingiu 170 respostas ao longo de todas as regiões brasileiras, com $36 \%$ referentes a estudantes e profissionais residentes no sul do Brasil, já as regiões com menor índice de respostas são o Norte e o Centro-Oeste, com 5\% e 6\%, respectivamente, como mostrado no gráfico 1 . Cerca de $63 \%$ dos entrevistados são do sexo masculino e $37 \%$ são do sexo feminino, tendo como base esses resultados, nota-se que o mercado da construção civil ainda tem tendência em possuir maior número de homens atuando na área. 
Gráfico 1 - Regiões que os entrevistados residem

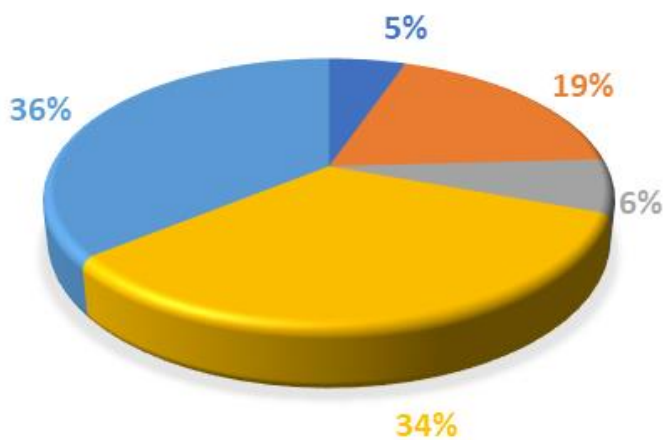

Norte

Nordeste

a Centro - Oeste

a Sudeste

国 Sul

Fonte - Os Autores

A faixa etária com maior número de entrevistados foi entre 25 a 34 anos de idade com $47,65 \%$ das respostas seguidas das faixas etárias de 18 a 24 anos $(34,71 \%)$ e 35 a 44 anos $(11,76 \%)$, como descrito no gráfico 2 . Dentre os entrevistados, cerca de $5,9 \%$ possui mestrado completo, $24,7 \%$ possuem especialização e cerca de $37 \%$ possuem graduação completa. Outros $17 \%$ são estudantes de graduação.

Gráfico 2 - Faixa etária dos entrevistados

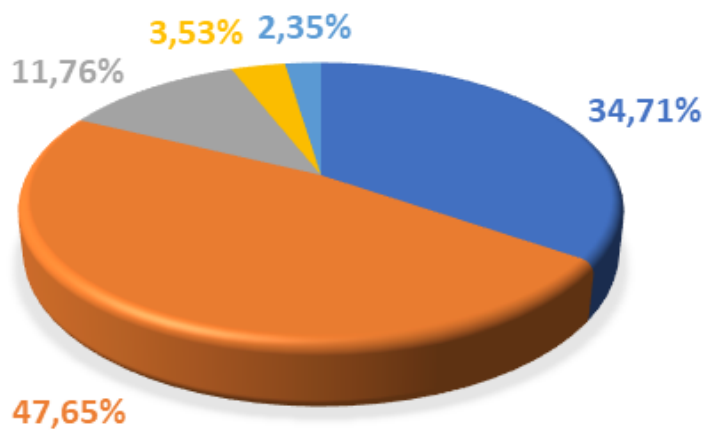

\author{
田 18 a 24 anos \\ Q 25 a 34 anos \\ 四35 a 44 anos \\ M 45 a 54 anos \\ 回55 a 64 anos \\ 田 65 anos ou mais
}

Fonte - Os Autores

Dentre as respostas obtidas, $50,6 \%$ tem formação em engenharia e $25,9 \%$ são graduandos do curso, na arquitetura, 15,8\% possuem formação e 4,1\% ainda são graduandos do curso, já a percentual restante se distribui em professores e profissionais de outras engenharias. $\mathrm{O}$ gráfico 3 mostra que o tempo de atuação dos profissionais entrevistados variaram entre recémformados e mais de 31 anos, sendo 41,8\% recém formados e 1,2\% de profissionais com 31 anos ou mais de atuação no mercado da construção civil.

Gráfico 3 - Tempo de atuação na profissão

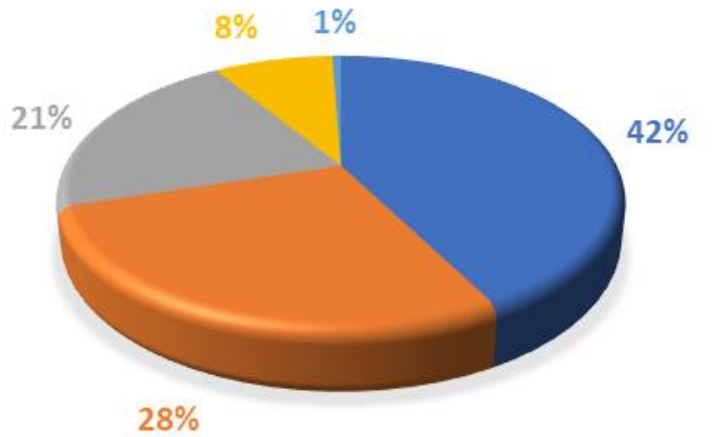

- Estudante/ Recém formado

国 1 a 3 anos

\4 a 10 anos

G11 a 20 anos

田21 a 30

Fonte - Os Autores 
Grande parte dos profissionais entrevistados atuam sozinhos, como profissionais liberais (cerca de 47,6\%) e a minoria atua em empresas de grande porte (com mais de 100 funcionários). Muitos profissionais desempenham mais de uma função na mesma empresa ou escritório e dentre as seguintes funções as que mais se destacam foram ser proprietário ou diretor da empresa e desenvolver projetos arquitetônicos, seguidas pelas funções de gerente de obras, gerente de projetos, e pelos responsáveis por elaboração de orçamentos e projetos estruturais, como representado no gráfico 4.

Gráfico 4 - Funções desempenhadas na empresa/escritório

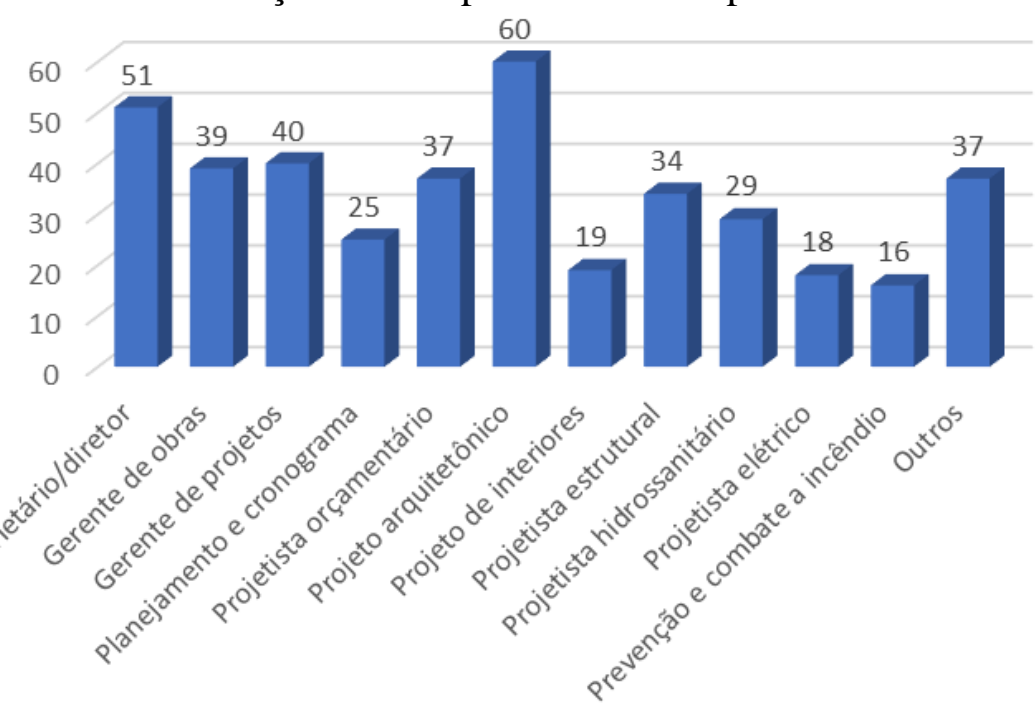

Fonte - Os Autores

A tecnologia BIM é conhecida por $87,6 \%$ dos entrevistados, no entanto, $0,6 \%$ não sabem o que é e $11,8 \%$ sabem que existe, mas não se interessaram em adquirir informações sobre esta tecnologia. A pesquisa apresentou que $7 \%$ dos escritórios em que os entrevistados trabalham não fazem uso de nenhum software BIM, 40,6\% não sabem dizer se o escritório usa ou não softwares BIM e os outros 52,4\% fazem uso para elaboração de projetos, compatibilização, orçamentos e quantitativo de materiais.

A utilização dos softwares BIM ainda está em progresso, não são todos os escritórios de engenharia e arquitetura que fazem uso, porém alguns não fazem uso porque é necessário tempo e organização a serem dedicados para o aprendizado dos funcionários sobre os novos softwares, e também pode ser por comodidade, já que os funcionários estão habituados a usarem os mesmos softwares que sempre foram utilizados no escritório, há casos em que não é utilizado pelo valor do software, como destacado no gráfico 5, outros motivos foram abordados e dentre eles, foi considerado desnecessário a utilização do BIM, por não ter uma alta demanda de projetos.

Gráfico 5 - Motivos da não utilização de softwares BIM

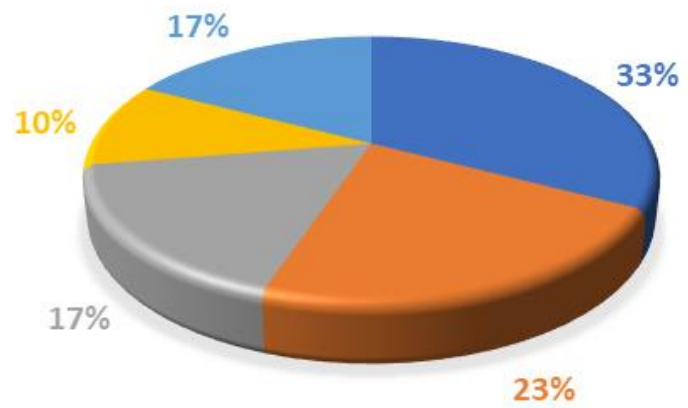

Fonte - Os Autores
G Valor do Software

Galta de tempo

Comodidade

Modernização dos equipamentos

GOutros 


\section{CONSIDERAÇÕES FINAIS}

A maioria dos entrevistados, cerca de $65,9 \%$, estão cientes de que a partir de janeiro de 2021, os projetos de obras públicas federais deverão ser projetados em softwares BIM, no entanto, quase metade dos entrevistados $(48,2 \%)$ ainda não sabia que a partir de janeiro de 2024, os softwares deverão estar presentes em projetos de obras públicas federais nas etapas de planejamento, orçamentação e informações de como construído, também conhecido como "as built".

A região Sul obteve $60 \%$ das suas respostas de profissionais e estudantes de engenharia, dentre esses, 50\% utilizam softwares BIM, enquanto dentre os profissionais e estudantes de arquitetura, que corresponderam a $40 \%$ desta região, $41 \%$ destes fazem uso dos softwares BIM nos escritórios em que trabalham. Nota-se que a região Sul possui mais integrantes da área de engenharia que utilizam o BIM do que da área de arquitetura, no entanto, a análise geral da região apresenta apenas $47 \%$ de todos os entrevistados, que operam esta tecnologia.

O Sudeste foi a região com o segundo maior número de respostas, e apresentou $92 \%$ dos entrevistados sendo estudantes e profissionais da área da engenharia. Entre eles, $51 \%$ fazem uso da tecnologia BIM nas empresas ou escritórios em que atuam. A parcela de entrevistados da área de arquitetura nesta região foi de apenas $8 \%$ e desses, apenas $25 \%$ utilizam o BIM, de forma geral, esta região permite observar que $51 \%$ das respostas foram positivas quanto ao uso da tecnologia.

As regiões Norte, Nordeste e Centro-Oeste, apresentaram menores índices de respostas, com $5 \%, 19 \%$ e $6 \%$ respectivamente, no entanto ambas apresentaram um índice maior na utilização dos softwares BIM entre os estudantes e profissionais que participaram da pesquisa, como demonstrado no gráfico 6 .

Gráfico 6 - Percentual de uso de softwares BIM por região brasileira

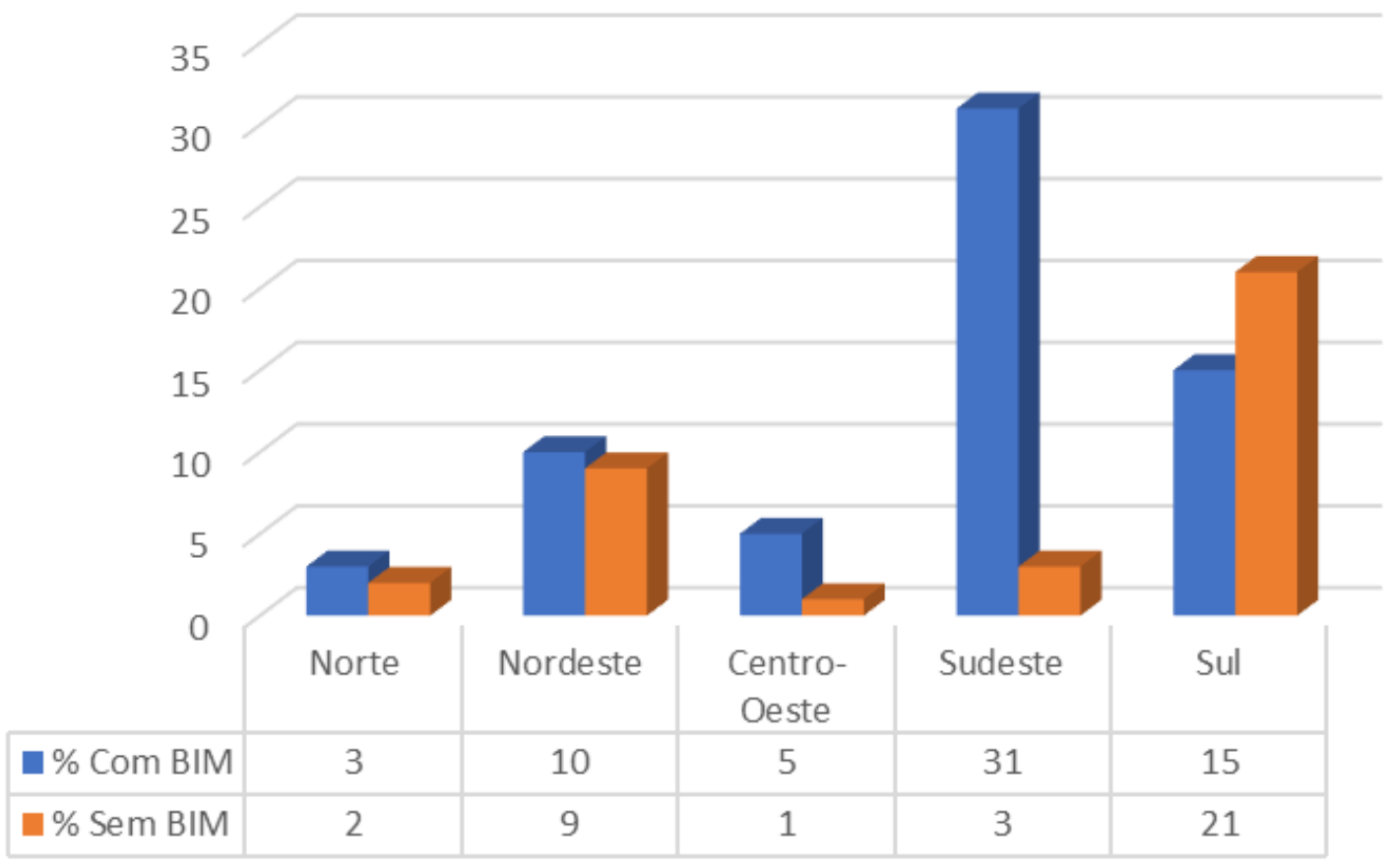

Fonte - Os Autores 
A obrigatoriedade imposta para as licitações de obras públicas federais, induz o mercado da construção civil a se modernizar como um todo, não somente na forma de construir, mas também na maneira em que os projetistas trabalham, pois estes precisam dedicar tempo, planejamento, empenho e investimento para acompanhar essa evolução de mercado e para ter como recompensa obras com menos contratempos que não foram previstos, tendo melhoras contínuas nos planejamentos.

De acordo com o IBGE (2020), a estimativa populacional do Brasil em 2020 é de aproximadamente 211 milhões de habitantes. Destes, 42,04\% residem na região Sudeste, $27,09 \%$ residem na região Nordeste, $14,26 \%$ na região Sul, $8,82 \%$ na região Norte e $7,79 \%$ na região Centro-Oeste. O número de respondentes do questionário ser maior na região Sul se explica pelo fato de as autoras residirem nesta região. Porém, a quantidade de respostas das outras regiões está proporcionalmente distribuída de acordo com a população de cada uma.

Em todo o país, o número de engenheiros civis que se encontram atualmente inscritos e ativos nos sistemas CREA/CONFEA é de 362.444, e o número de arquitetos com o cadastro ativo no CAU é de 188.645. De acordo com o MEC (2017), existem 346.827 estudantes matriculados nos cursos de engenharia civil e 168.291 nos cursos de arquitetura distribuídos pelo país. Portanto, para esta população de 1.066.207, e para o tamanho da amostra obtida (170 respostas), tem-se uma pesquisa com confiabilidade de $95 \%$ e margem de erro de 7,5\%.

Sabe-se que a amostra obtida na presente pesquisa representa apenas uma parcela dos profissionais e estudantes de todo o país. Porém, apesar da pequena amostragem, os resultados obtidos podem representar um diagnóstico em relação à atual disseminação da tecnologia BIM e a perspectiva de futuro entre profissionais e estudantes de engenharia e arquitetura tanto para a elaboração, quanto para a compatibilização, planejamento, gerenciamento e controle de projetos no segmento da construção civil.

A adoção de BIM já é uma realidade, e entendida como um processo sem volta. Assim como a mudança do papel e prancheta para ferramentas CAD de desenho através do computador, o BIM também veio para ficar e, mais cedo ou mais tarde irá se tornar padrão entre projetos de edifícios.

\section{AGRADECIMENTOS}

Nossos sinceros agradecimentos a todos os 170 profissionais e estudantes de engenharia e arquitetura que se dedicaram a responder o questionário e a contribuir para o desenvolvimento deste estudo. Ao todo, foram 62 participantes da região Sul, 56 da região Sudeste, 32 da região Nordeste, 11 da região Centro-oeste e 9 da região Norte. 


\section{REFERÊNCIAS BIBLIOGRÁFICAS}

ASSOCIAÇÃO BRASILEIRA DE NORMAS TÉCNICAS. NBR 14724: informação e documentação - trabalhos acadêmicos - apresentação. Rio de Janeiro, 2011.

Janeiro, 2002.

NBR 6023: informação e documentação - referências - elaboração. Rio de

BIBLUS. As dimensões do BIM: 3D, 4D, 5D, 6D, 7D. Biblus, 2018. Disponível em: $<$ http://biblus.accasoftware.com/ptb/as-dimensoes-do-bim-3d-4d-5d-6d-7d/>. Acesso em: 28 Julho 2020.

BRASIL. Decreto ${ }^{\circ}$ 9.377. Dispõe sobre a Estratégia Nacional de Disseminação do Building Information Modelling e institui o Comitê Gestor da Estratégia do Building Information Modelling. Brasília, DF, 22 ago 2018.

Decreto $n^{\circ}$ 9.983. Dispõe sobre a Estratégia Nacional de Disseminação do Building Information Modelling e institui o Comitê Gestor da Estratégia do Building Information Modelling. Brasília, DF, 22 ago 2019.

Decreto $\mathrm{n}^{\circ}$ 10.306. Estabelece a utilização do Building Information Modelling na execução direta ou indireta de obras e serviços de engenharia realizada pelos órgãos e pelas entidades da administração pública federal, no âmbito da Estratégia Nacional de Disseminação do Building Information Modelling. Brasília, DF, 02 abr 2020.

Ministério da Economia. Building Information Modelling - BIM, 2018. Disponível em: <http://www.mdic.gov.br/index.php/competitividade-industrial/ce-bim>. Acesso em: 28 Jul 2020.

Ministério da Educação. Censo da Educação Superior 2017. Brasília, DF, 2018.

Disponível em: < http://portal.mec.gov.br/docman/setembro-2018-pdf/97041-apresentac-a-ocenso-superior-u-ltimo/fil >. Acesso em: 08 Set 2020.

IBGE - Instituto Brasileiro de Geografia e Estatística. Estimativas de população publicadas no DOU: Tabelas de estimativas populacionais para os municípios e para as Unidades da Federação brasileiros em 01.07.2020. Disponível em:

$<$ https://www.ibge.gov.br/estatisticas/sociais/populacao/9103-estimativas-de-

populacao.html?=\&t=resultados $>$. Acesso em: 08 Set 2020.

PEREIRA, P. A. I; RIBEIRO, R. A. A inserção do BIM no curso de graduação em Engenharia Civil, 2015. Disponível em:

<https://www.researchgate.net/publication/290434979_A_insercao_do_BIM_no_curso_de_gr aduacao_em_Engenharia_Civil>. Acesso em: 28 jul 2020.

SEIL - Secretaria de Estado de Infraestrutura e Logística. Caderno BIM: coletânea de cadernos orientadores: caderno de especificações técnicas para contratação e projetos em BIM - Edificações. Coordenação: Lucimara Ferreira de Lima. Curitiba, PR, 2018. 